\title{
Electrical Studies of Barkhausen Switching Noise in Ferroelectric PZT: Critical Exponents and Temperature Dependence
}

\author{
C. D. Tan, ${ }^{1}$ C. Flannigan, ${ }^{1}$ J. Gardner, ${ }^{2}$ F. D. Morrison, ${ }^{2}$ E. K. H. Salje, ${ }^{3,4}$ and J. F. Scott ${ }^{1,2}$ \\ ${ }^{1}$ School of Physics and Astronomy, \\ University of St Andrews, St Andrews, Fife KY16 9SS, UK \\ ${ }^{2}$ School of Chemistry, University of St Andrews, St Andrews, Fife KY16 9ST, UK \\ ${ }^{3}$ Dept. Earth Sciences, Cambridge Univ., Cambridge CB2 3EQ, UK \\ ${ }^{4}$ State Key Laboratory for Mechanical Behavior of Materials, \\ Xi'an Jiaotong University, Xi'an 710049, China
}

\begin{abstract}
Crackling noise of ferroelectric lead zirconate titanate (PZT) samples during ferroelectric switching is demonstrated to be compatible with avalanche statistics. The peaks of the slew-rate (time derivative of current $d I / d t$ squared), defined as "jerks", were statistically analyzed and shown to obey power-laws. The critical exponent obtained is $1.64 \pm 0.15$, in agreement with predictions from avalanche theory. The exponent is independent of temperature within experimental error margins.
\end{abstract}

\section{INTRODUCTION}

A complete analysis of the dynamic properties of ferroelectric domain switching [1] showed that the statistical switching variables (switching amplitudes, energy, and interevent times) in $\mathrm{BaTiO}_{3}$ follow avalanche statistics. The switching exponents are universal and close to values of the field integrated mean field model, which predicts that the stressintegrated

distribution of pulse energies scales as

$$
p(E) \sim E^{-5 / 3}=E^{-1.67}
$$

[2]. The experimental approach in [1] was to measure the acoustic emission, AE, generated by moving 90-degree boundaries in $\mathrm{BaTiO}_{3}$. Such $\mathrm{AE}$ distributions have been observed previously during ferroelastic phase transitions [2-5] and their origin was identified as moving domains [6]. Here we show that the same criticality is seen by purely electronic 
measurements in the most common ferroelectric material (lead zirconate titanate, PZT). Small jumps in the ferroelectric hysteresis curve are identified as being due to moving domains. These jumps occur at the steepest region of the polarization hysteresis curve [7, 8]. When electric fields are applied, the domains initially aligned antiparallel to the field will reverse creating interfaces, domain walls $[9,10]$, extending through space. When the domain walls move, they may come across regions near defects and become pinned $[7,8]$. As the magnitude of the field increases, the domain walls will eventually gain enough energy to overcome the pinning and move forward, causing an increase in polarization [7, 8]. The rapid nucleation of new domain walls and their 'jerky' movements are responsible for the temporal changes in polarization.

Barkhausen noise and many other crepitations are statistically similar: they resemble avalanches and the size distribution of these crackling noises obeys a power-law with characteristic exponents [6,11-14]. Due to universality, these exponents allow avalanche systems to be compared with one another, leading to a lot of interesting physics being unveiled [6, 11, 12]. For example, Dahmen and Ben-Zion (2009) in [12] showed that Barkhausen noise and earthquake events share many statistical similarities. Meanwhile, Baró et al. (2013) in [15] demonstrated that earthquakes and compressions of porous materials are statistically alike too. Performing these experiments under the safety of the laboratory allows researchers to learn more about seismology and improve earthquake risk assessments $[6,16,17]$.

The investigation of purely electrical noise is a very important problem in low electric fields for PZT (lead zirconate titanate) for medical MRI imaging [18] and in high electric fields [19] for switching devices such as ferroelectric memory cards ( $\$ 100$ million/year product last year for subway fare cards, etc., in Japan and the USA). However, most reviews are limited to the low-field situation [20]. Two commercial PZT ceramics, PIC 151 and PIC 255, from PI Ceramic Lederhose, Germany [21] were categorized using a conventional hysteresis apparatus and the jerky changes in the polarization switching current responses were shown to obey power-laws (critical exponents listed in Table I below). If treated as proxies for energy exponents, our results are in agreement with predictions of avalanche theory [6, 14, 22-25]. The actual compositions of both samples are proprietary and 
therefore undisclosed; but from the product brochure [21], PIC 255 was designed to have a higher coercive field $E_{c}$ compared to PIC 151.

In addition to the basic critical exponent measurement, we have explored temperature dependence of the crackling noise. We find that as temperature is increased towards the Curie temperature $T_{c}$, there is a non-monotonic evolution of a second exponent, reaching a peak at ca. 1.90 at $\mathrm{T}=413 \mathrm{~K}$. This effect may be related to the dynamical behaviour of dislocations. PZT has a large number [26-28] of threading dislocations $\left(10^{13} \mathrm{~cm}^{-2}\right.$ at best, about an order of magnitude greater than in $\mathrm{BaTiO}_{3}$ ), and empirical evidence from avalanche in other systems (such as porous metals) suggests that exponents near 1.9-2.0 are due to such dislocations [29].

\section{EXPERIMENT DETAILS AND PROCEDURES}

The measurements were conducted using an aixACCT TF Analyzer $2000[30,31]$ with a high voltage set-up that allows voltage pulses ranging from $200 \mathrm{~V}$ to $40 \mathrm{kV}$ [31] to be applied to the sample via the probes.

In our experiments, triangular electric field/voltage pulses were designed using a manual waveform generator from the TF Analyzer 2000 software by providing the target voltage values at specific time-stamps. As the coercive field $E_{c}$ of ferroelectrics changes with the voltage ramp-rate [32,33], the time taken for different maximum voltages were calculated

and specified to keep the ramp rate constant. The ramp rate was $40 \mathrm{Vs}^{-1}$ for PIC 151 and 60 $\mathrm{Vs}^{-1}$ for PIC 255. A down-switching-pulse was applied through a sample via the probes followed by an equal and opposite up-switching-pulse. The current-time response during the linear ramping region of the up-switching-pulse was then acquired. Due to the limitations of the apparatus, the highest achievable sampling rate was $40 \mathrm{~Hz}(1000$ points 25 seconds). 


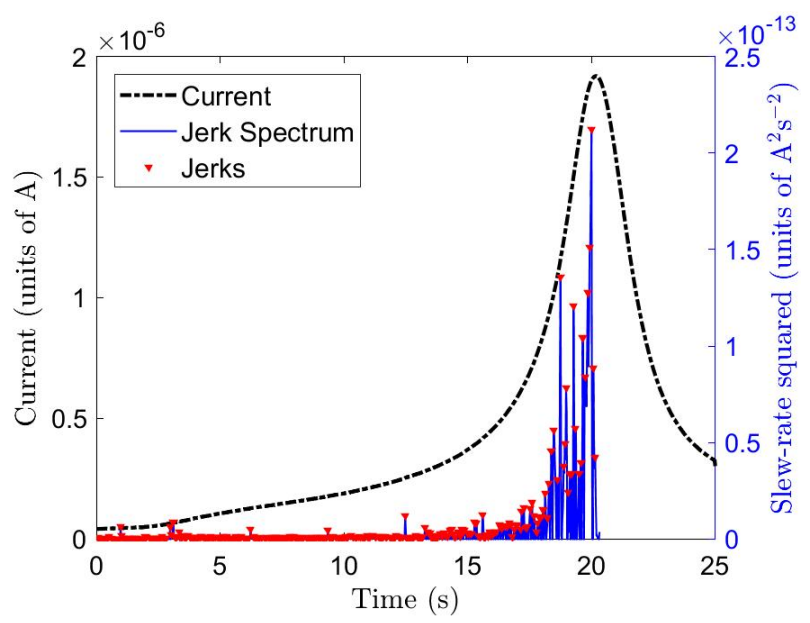

Figure 1. (color online) A single measurement from PIC 151. The smooth looking current I curve (black dashed) was jerky, as shown after taking the square of its time derivative, (dI/dt)2 and removing the baseline (blue). The peaks (red labeled) were used for statistical analysis. The baseline removed graph is conventionally called the jerk spectrum.

Figure 1 shows the current $I$ response (black dashed) of PIC 151 against time. The measured current is not smooth but consists of small jumps called jerks and may indicate very small events [6]. Procedures for extracting the jerks from the measured current $I$ were as follows:

1. The first time derivative $d I / d t$ is taken and squared, revealing the jerky nature of the current response with peaks superposed onto a smooth baseline.

2. The minima were then fitted using the Piecewise Cubic Hermite Interpolating Polynomial (PCHIP) function from Matlab R.

3. The fitted line was subtracted, leaving $(d I / d t)^{2}$ with its baseline removed (blue solid in Figure 1). This is conventionally called the jerk spectrum [6].

4. The peaks in the jerk spectrum (red labels in Figure 1) are defined as jerks $J$ and are subjected to statistical analyses.

In avalanche studies, jerks can be defined in multiple ways [6, 23, 34]. In He et al. (2016) [34] ferroelastic switching simulations, the jerks were defined as the total change in potential energy, the energy drop and the shear stress drop. In crystal plasticity 
experiments, the jerks were defined as the square of the velocity $v$ of slip avalanches squared, $(d v / d t)^{2}$, which took the form of an energy [6, 14, 22, 25].

In our case, the jerks are defined as peaks of the square of the slew-rate [35], $(d I / d t)^{2}$. The slew-rate $d I / d t$ is a term commonly found in electronics to describe how fast an input waveform can change when it passes through an op-amp before the signal is distorted [36]. The slew-rate is usually defined as the time derivative of voltage $d V / d t$ [36] but it can also be defined as $d I / d t$ [35]. Taking the square of the slew-rate allows the peaks in our jerk spectrum to closely represent the jerks defined in crystal plasticity and acoustic emission experiments, which is $(d v / d t)^{2}$ as mentioned. In this study, we successfully show that $J$ extracted from the slew-rate obeys a power-law $[6,11,12]$ :

$$
P(J)=\frac{\varepsilon-1}{J_{\min }}\left(\frac{J}{J_{\min }}\right)^{-\varepsilon}
$$

where $\varepsilon$ is an exponent and $J_{\min }$ is the lower-bound normalization condition $[37,38]$.

\section{RESULTS AND DISCUSSION}

A total of five measurements were taken for both PIC 151 and PIC 255. For each sample individually, the jerk peaks were extracted, normalized by the mean and combined for statistical evaluation. A total of 1261 peaks were retrieved for PIC 151 and 1468 peaks for PIC 255.

The peaks for both samples were logarithmically binned and the distributions were fitted with two straight lines (Figure 2). The slope yields -1.59 for PIC 151 (blue circles) and -1.50 for PIC 255 (red squares).

Following [37], high coefficient of determination $R^{2}$ value of the linear fit cannot be trusted. Non-power-law distributed histograms can resemble a power-law distribution over many orders of magnitude, thus providing a large $R^{2}$ value. Also, the jerk data a priori could involve two exponents or more (or a whole Gaussian distribution of exponents). Fitting a straight-line could lead to a false slope that was due to the averaging effect of exponents [38]. And lastly, the starting and ending limits of the fitted data should be independently

justified. The data in Figure 2 clearly demonstrate a straight-line behavior. Nevertheless, it is desirable to show that this is robust, and that the apparent power law distribution corresponds to a single exponent $[37,38]$. 
To accomplish these buttressing arguments we utilize the Maximum Likelihood (ML) method. This is a statistically reliable way of demonstrating a single exponent in $\varepsilon$ power law dependence. The procedure is sketched below and provided in complete detail elsewhere [38].

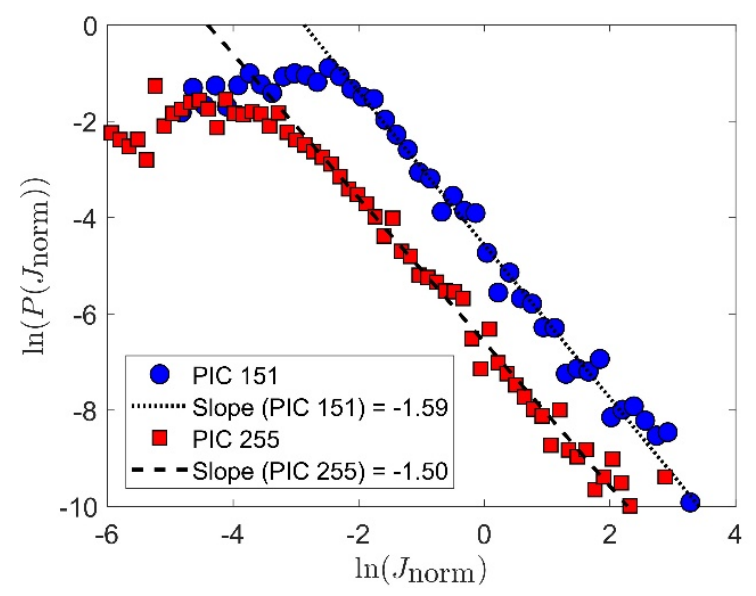

Figure 2. (color online) The log-log probability distribution $P\left(J_{\text {norm }}\right)$ of normalized jerks $J_{\text {norm }}$ from PIC 151 (blue circles) and PIC 255 (red squares) shows a straight-line behavior. The slope is linearly fitted at region $\ln \left(\right.$ Jnorm $_{\text {) }}>-2$ for PIC 151 is -1.59 and for PIC 255 is -1.50 .

ML estimates how likely a scaling parameter, the exponent $\varepsilon$ from the power-law model, had generated an experimental jerk spectrum [37, 39]. The estimated exponent derived [37] from maximizing the likelihood function with respect to an exponent $\varepsilon$ is:

$$
\hat{\varepsilon}=1+N\left[\sum_{i=1}^{N} \ln \frac{J_{i}}{J_{\min }}\right]^{-1}
$$

where $\hat{\varepsilon}$ is the convention used to denote that the exponent is an estimate [37-39]. The standard error of $\hat{\varepsilon}$ is:

$$
\text { Standard error, } \sigma=\frac{\hat{\varepsilon}-1}{\sqrt{N}}+\text { higher-order terms }
$$

where the higher-order terms are positive [37].

Not all data points are power-law distributed in experimental settings. Due to the limitations of the apparatus, low signals may be under-counted due to saturation effects [38] which leads to an unknown lower bound $J_{\min }$ in eq. (3) [37, 38]. By guessing the value of $J_{\min }$ and discarding any jerk data $J$ less than $J_{\min }$, one runs the risk of [37]: 
1. Underestimating $J_{\min }$, performing a power-law ML estimate to non-power-law $J$ data.

2. Overestimating $J_{\min }$, discarding valid data points and increasing the statistical error of $\hat{\varepsilon}$.

Thus to fully utilize the ML estimation, a computational algorithm of eq. (3) is iterated on the jerk data by a series of cut-off values $J_{0}$ (that replaces $J_{\min }$ ) [37, 38]. For each $J_{0}$ value, data points less than $J_{0}$ are discarded and an estimated exponent $\hat{\varepsilon}$ is computed. This results in an array of $\hat{\varepsilon}$ which is plotted against the natural-log transformed $\ln \left(U_{0}\right.$ ) (see Figure 3 ). This method is called lower-bound estimation [37] but is conventionally understood as performing the Maximum Likelihood Estimation/Method/Fit [6, 16, 38, 40]. The end result is a graph that has a broad plateau at the most-likely exponent, which starts with a kink at the correct $J_{0}=J_{\min }$ value and extends for a range of $J_{0}[37,38]$. The plateau then fluctuates at large $J_{0}$ values and the error bar increases due to a lack of statistically relevant data points [38]. If there is no plateau, the assumption of a single-exponent power law is invalid.

In experimental settings, ML curves and log-log binning plots are the keys to understanding an avalanche system. Many subtleties of a system can be uncovered using ML analysis, which is described in [38].

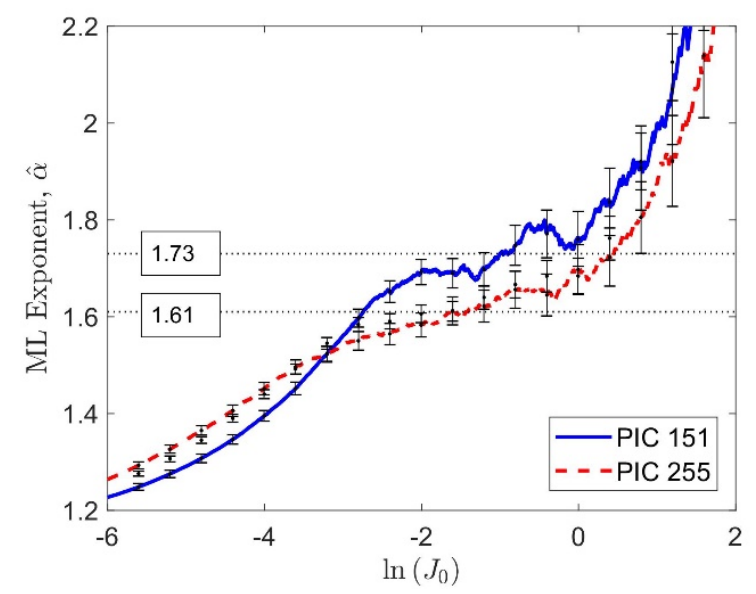

Figure 3. (color online) Maximum-likelihood (ML) fit of PIC 151 (blue solid) and PIC 255 (red dashed). PIC 151 displays a plateau of two decades at $-2.0 \leq \ln (\mathrm{J} 0) \leq 0.0$ with an average estimated exponent $\hat{\varepsilon}$ of 1.73 . PIC 255 shows a plateau of three decades at $-3.0 \leq \ln \left(\mathrm{J}_{0}\right) \leq 0.0$ with $\hat{\varepsilon}$ of 1.61 . Both averaged exponents have a standard deviation $\sigma$ of \pm 0.04 . The lack of definitive kinks at $J_{0}=J_{\min }$ for both $M L$ curves was due to lower cut-off effects that arose from saturation effects in the measurement apparatus [38].

The jerk peaks J for PIC 151 and PIC 255 were subjected to ML analyses (see Figure 3). PIC 151 (blue solid curve) exhibits a plateau of two decades at $-2.0 \leq \ln \left(J_{0}\right) \leq 0.0$ and the 
averaged exponent $\hat{\varepsilon}$ at this region yields 1.73. The plateau for PIC 255 (red dashed) extends for three decades at $-3.0 \leq \ln \left(J_{0}\right) \leq 0.0$ with an averaged exponent $\hat{\varepsilon}$ of 1.61 . The standard deviations $\sigma$ for both averaged exponents are \pm 0.04 .

The values for both $\hat{\varepsilon}$ and $\sigma$ for each ML analysis depended highly on where the plateaus were defined and would be biased. Unlike ideal power-laws, there were no definitive kinks to determine the value of $J_{\min }$ and where the plateau started due to the lower cut-off effect [38]. The lower cut-off effect is also observed in the two jerk distributions in Figure 2. The straight-line behavior extended until $\ln \left(U_{\text {norm }}\right) \approx-2$ and followed by a smooth cut-off due to saturation effects [38].

An additional PIC 151 sample (151b) was studied and a total of 2722 peaks were extracted. The jerk spectrum exhibits intense peaks that are at least one magnitude larger than the usual jerk peaks seen in the former two samples and may represent spanning avalanches [34]. As mentioned by He et al. (2016) in [34], these peaks do not play a role in avalanche statistics and 41 peaks above an arbitrarily set cut-off value were removed. The ML estimate over the remaining 2681 jerk data yields an exponent $\hat{\varepsilon}$ of 1.64 with $\sigma=0.04$ when averaged over a plateau at region $-2.7 \leq \ln \left(U_{0}\right) \leq 0.0$.

Statistical noise from electronic systems will influence the ML curve, causing an increase in the measured exponent. Thus, based on both the linear fit of the log-log power law distribution and the ML curve, we estimated that the exponent should be $1.64 \pm 0.15$ to take the random electrical noise effects into account.

\begin{tabular}{|c|c|c|c|c|}
\hline \multirow{2}{*}{ Systems } & PIC 151 & PIC 255 & PIC 151b & Field-driven MFT \\
\hline$\varepsilon$ & & & & \\
$\varepsilon$ & $1.73 \pm 0.04$ & $1.61 \pm 0.04$ & $1.64 \pm 0.04$ & 1.67 \\
\hline
\end{tabular}

Table I. Summarized critical exponents $\varepsilon$ of PIC 151, PIC 255 and PIC 151b from this study and fielddriven energy exponent from mean field theory (MFT) [6].

Existing avalanche theory makes no direct prediction about the temperature dependence of Barkhausen pulse dynamics besides the invariance of the mean field results. However, we may expect that these dynamics change at ultra-low temperatures where creep freezes out and is replaced by quantum tunneling of domain walls [41]; and at high 
temperatures near $\mathrm{T}_{\mathrm{c}}$ the wall mobility increases dramatically and domain sizes decrease which may potentially lead to deviations from the mean field behavior.

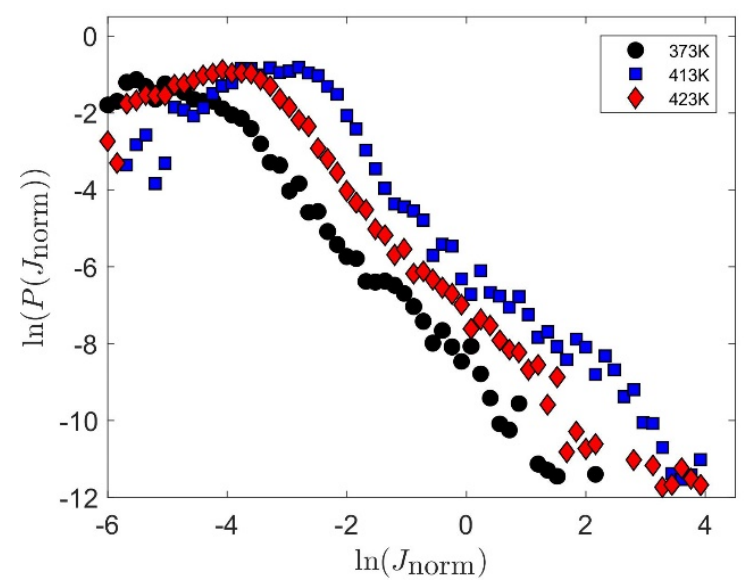

Figure 4. (color online) $P(J)$ versus J for three different temperatures, showing power-law mixing at temperatures near $413 \mathrm{~K}$ $\left(140{ }^{\circ} \mathrm{C}\right)$. An attempt to fit these distributions with a straight-line will lead to an averaging effect of two exponents [38].

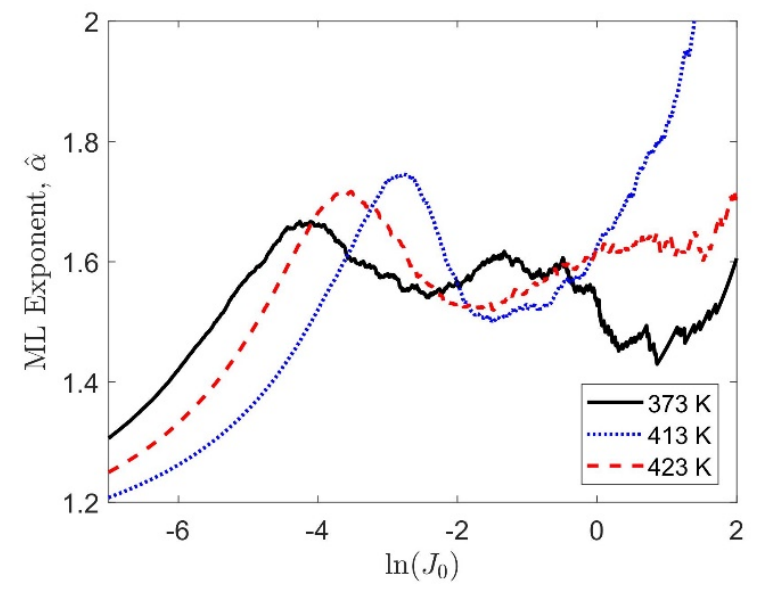

Figure 5. Maximum likelihood graphs at three different temperatures, verifying the power-law mixing effect from the slopes in Figure 4. The initial kinks of the ML curves underestimate the first critical exponent, they then decrease to the value of the second critical exponent.

Figure 4 shows the behavior of Barkhausen dynamics as $\mathrm{T}$ is increased towards $\mathrm{T}_{\mathrm{c}}$. At ca. $\mathrm{T}=410 \mathrm{~K}$, the straight-line behavior of the power-law exponent 1.65 for $P(J)$ against $J$ gives way to a superposition of two separate exponential distributions, one of which is greater at $1.80-2.00$.

The maximum likelihood analysis reveals the power-law mixing effect of two simultaneous exponents. From Figure 5, the initial kinks of the ML curves approach but underestimate the first exponent. They then decrease to the value of the second lower 
exponent before fluctuating due to a lack of statistically relevant data. In other words, the jerk peaks extracted from higher temperatures were generated from two different powerlaw mechanisms. The second, smaller exponent remains near 1.6 and is independent of temperature.

We suggest an explanation for this: PZT is known to have a very high density of dislocations (ca. $10^{13} \mathrm{~cm}^{-2}$ ) even in very good samples, about an order of magnitude greater than in $\mathrm{BaTiO}_{3}$, and neighboring dislocations ca. $12 \mathrm{~nm}$ apart [26, 27]. These are mostly threading dislocations[28].

It is known empirically that de-pinning from dislocations gives an exponent near 2.0 [29]. Our tentative hypothesis for the second, higher exponent is the passage through the temperature region in which ferroelectric domain walls de-pin from threading dislocations. De-pinning temperatures significantly below $T_{c}$ are well known in other ferroelectrics: $\mathrm{CsD}_{2} \mathrm{AsO}_{4}[42,43]$. In magnets, Bohn et al. have demonstrated [44-46] different universality classes in Barkhausen pulses. These emphasize dimensionality as well as thicknesses of domains and explicitly include exponent crossover as fields or temperature increases, domains become smaller, and de-pinning becomes different from occasional creep. This could give rise to two exponents. However, the comparison of both hard and soft PZT in the present work makes this hypothesis unlikely in our case. Most importantly, the switching exponent $\varepsilon=1.64$ remains independent of temperature.

\section{CONCLUSION}

From two commercial PZT samples albeit with a low sampling rate, we showed that the jerks obeyed power-law statistics and were consistent with Barkhausen noise [6,12]. The distribution of Barkhausen noises obeys a power-law and the determined exponents with an estimated value of $1.64 \pm 0.15$ are in agreement with values from avalanche theory.

This observation, when compared with the expected MFT exponent 1.33 [6, 14, 22-25] for single avalanches and 1.67 for field-integrated models, indicates that the polarization switching follows a field-integrated pathway. This indicates that after each pinning or depinning event an exponential relaxation follows as part of the avalanche. We conclude that the switching processes in our PZT samples take the form of complex avalanches and are consistent with Barkhausen noise and many other crackling systems. The discovery 
and categorization of these exponents are crucial as they show that the domain switching mechanisms in ferroelectric ceramics demonstrate criticality $[2,47,48]$. Other exponents for the amplitudes and, most importantly, for the inter-event times for domain switches, cannot be obtained from our measurements. Nevertheless, these parameters were measured for the domain switching in $\mathrm{BaTiO}_{3}$ and we may assume that the values in PZT follow those of $\mathrm{BaTiO}_{3}$ very closely. Future work on the acoustic emission in PZT is highly desirable to identify such full sets of switching parameters and to compare with other ferroelectric materials.

\section{ACKNOWLEDGMENTS}

This work at St Andrews was supported by EPSRC grant EP/P024637/1. EKHS thanks EPSRC for support.

[1] E. K. H. Salje, D. Xue, X. Ding, K. A. Dahmen, and J. F. Scott, Physical Review Materials in press.

[2] P. Bak, C. Tang, and K. Wiesenfeld, Physical Review A 38, 364 (1988).

[3] E. K. H. Salje, X. Ding, Z. Zhao, T. Lookman, and A. Saxena, Physical Review B 83, 104109 (2011).

[4] R. J. Harrison and E. K. H. Salje, Applied Physics Letters 97, 021907 (2010).

[5] S. Puchberger, V. Soprunyuk, W. Schranz, A. Tr"oster, K. Roleder, A. Majchrowski, M. A. Carpenter, and E. Salje, APL Materials 5, 046102 (2017).

[6] E. K. H. Salje and K. A. Dahmen, Annual Review of Condensed Matter Physics 5, 233 (2014).

[7] V. M. Rudyak, Soviet Physics Uspekhi 13, 461 (1971).

[8] R. Tebble, IOPScience 2, 1017 (1955).

[9] E. K. H. Salje, ChemPhysChem 11, 940 (2010).

[10] D. Damjanovic, Reports on Progress in Physics 61, 1267 (1998).

[11] J. P. Sethna, K. A. Dahmen, and C. R. Myers, Nature 410, 242 (2001). 
[12] K. A. Dahmen and Y. Ben-Zion, in Encyclopedia of Complexity and Systems Science (Springer New York, New York, NY, 2009) pp. 5021-5037.

[13] K. Dahmen and J. P. Sethna, Physical Review B 53, 14872 (1996).

[14] K. A. Dahmen, in Avalanches in Functional Materials and Geophysics, Understanding Complex Systems, edited by E. K. Salje, A. Saxena, and A. Planes (Springer International Publishing, Cham, 2017) pp. 19-30.

[15] J. Bar'o, A. Corral, X. Illa, A. Planes, E. K. H. Salje, W. Schranz, D. E. Soto-Parra,' and E. Vives, Physical Review Letters 110, 088702 (2013).

[16] X. Jiang, H. Liu, I. G. Main, and E. K. Salje, Physical Review E 96, 1 (2017).

[17] E. K. H. Salje, American Mineralogist 100, 343 (2015).

[18] R. L. Goldberg and S. W. Smith, Ultrasonic Imaging 17, 95 (1995).

[19] N. C. Pisenti, A. Restelli, B. J. Reschovsky, D. S. Barker, and G. K. Campbell, Review of Scientific Instruments 87, 124702 (2016).

[20] M. Sayer, C. V. R. V. Kumar, F. V. Letelier, J. Hazell, M. Watt, and P. Donders, Integrated Ferroelectrics 5, 79 (1994).

[21] "Piezoelectric Ceramic Products Brochure by PI ceramic GmbH Available: https://www.piceramic.com/en/products/piezoceramic-materials. Accessed 21 April 2018,".

[22] N. Friedman, A. T. Jennings, G. Tsekenis, J.-Y. Kim, M. Tao, J. T. Uhl, J. R. Greer, and K. A. Dahmen, Physical Review Letters 109, 095507 (2012).

[23] Y. Ben-Zion, K. A. Dahmen, and J. T. Uhl, Pure and Applied Geophysics 168, 2221 (2011). [24] K. A. Dahmen, Y. Ben-Zion, and J. T. Uhl, Physical Review Letters 102, 175501 (2009).

[25] G. Tsekenis, J. T. Uhl, N. Goldenfeld, and K. A. Dahmen, EPL (Europhysics Letters) 101, 36003 (2013).

[26] V. Nagarajan, C. L. Jia, H. Kohlstedt, R. Waser, I. B. Misirlioglu, S. P. Alpay, and R. Ramesh, Applied Physics Letters 86, 192910 (2005).

[27] C. L. Canedy, H. Li, S. P. Alpay, L. Salamanca-Riba, A. L. Roytburd, and R. Ramesh, Applied Physics Letters 77, 1695 (2000).

[28] I. Vrejoiu, G. Le Rhun, N. D. Zakharov, D. Hesse, L. Pintilie, and M. Alexe, Philosophical Magazine 86, 4477 (2006). 
[29] P. O. Castillo-Villa, J. Bar'o, A. Planes, E. K. H. Salje, P. Sellappan, W. M. Kriven, and E. Vives, Journal of Physics: Condensed Matter 25, 292202 (2013).

[30] aixACCT - TF Analyzer 2000. Available: http://www.aixacct.com/html/prod/memprods tf2000.php,

Accessed 23 January 2018.

[31] TF Analyzer 2000 Hysteresis Software, 2nd ed. (aixACCT Systems GmbH, Aachen) p. 174.

[32] J. F. Scott, Integrated Ferroelectrics 12, 71 (1996).

[33] D. Viehland and Y. H. Chen, Journal of Applied Physics 88, 6696 (2000).

[34] X. He, X. Ding, J. Sun, and E. K. H. Salje, Applied Physics Letters 108, 072904 (2016).

[35] A. Charalambous, X. Yuan, and N. McNeill, IEEE Transactions on Power Electronics 33, 5660 (2018).

[36] B. Carter, in Op Amps for Everyone (Elsevier, Oxford, 2013) pp. 195-223.

[37] A. Clauset, C. R. Shalizi, and M. E. J. Newman, SIAM Review 51, 661 (2009).

[38] E. K. H. Salje, A. Planes, and E. Vives, Physical Review E 96, 042122 (2017).

[39] J. A. Rice, Mathematical Statistics and Data Analysis, 3rd ed. (Thomson Brooks/Cole, Belmont, CA, 2007) p. 688.

[40] M. C. Gallardo, J. Manchado, F. J. Romero, J. Del Cerro, E. K. Salje, A. Planes, E. Vives, R. Romero, and M. Stipcich, Physical Review B - Condensed Matter and Materials Physics 81, 1 (2010).

[41] F. Kagawa, S. Horiuchi, and Y. Tokura, Crystals 7, 106 (2017).

[42] A. A. Baski, W. F. Oliver, and J. F. Scott, Ferroelectrics Letters Section 7, 171 (1987).

[43] A. Sidorkin, N. Burdanina, and L. Kamysheva, Soviet Physics-Solid State 26, 1910 (1984).

[44] F. Bohn, G. Durin, M. A. Correa, N. R. Machado, R. D. Della Pace, C. Chesman, and R. L. Sommer, Scientific Reports 8, 11294 (2018).

[45] G. Z. d. S. Lima, G. Corso, M. A. Correa, R. L. Sommer, P. C. Ivanov, and F. Bohn, Physical Review E 96, 022159 (2017).

[46] A. P. Mehta, A. C. Mills, K. A. Dahmen, and J. P. Sethna, Physical Review E 65, 046139 (2002).

[47] P. Bak, C. Tang, and K. Wiesenfeld, Physical Review Letters 59, 381 (1987).

[48] P. Bak and K. Chen, Scientific American 264, 46 (1991). 